\title{
De secuestros y localizaciones de niños y niñas "desaparecidos/as": los albores del activismo de Abuelas de Plaza de Mayo
}

\author{
SABINA A. REGUEIRO
}

\begin{abstract}
Resumen
Este artículo analiza las incipientes formas de activismo de un grupo de mujeres, las Abuelas de Plaza de Mayo, cuyo objetivo es la búsqueda de niños/as desaparecidos/as durante la última dictadura militar argentina. Para iluminar este proceso se reflexionará sobre la primera localización de un niño y una niña acompañada por "Abuelas". Tras el secuestro de sus padres, dos hermanos son "abandonados" en una plaza pública, internados en un instituto de menores y entregados en guarda a una pareja. Sin embargo, la búsqueda de sus abuelas en toda la región permitió su localización un año después. Se desató así un conflicto público entre las familias en disputa en torno a la legitimidad de la adopción o la restitución. Esto habilitó la intervención tanto de autoridades militares desde las embajadas involucradas como de diversos representantes locales de organismos internacionales en el marco de un debate mediático, político y regional en torno a la "subversión". De esta manera, a partir de este estudio de caso, se contribuirá al debate general en torno a la compleja relación entre política, derechos, parentesco e infancia.
\end{abstract}

Palabras clave: Abuelas de Plaza de Mayo - Activismo político de mujeres - Adopción - Parentescos - Infancia

\begin{abstract}
This articule analizes the incipient forms of activism of a group of women, "Abuelas de Plaza de Mayo", whose goal was the search of children dissappeared during the last military dictatorship in Argentina. To enlighten this process we will think over the first location of a girl and a boy by "Abuelas". After the kidnapping of their parents, two siblings are "abandoned" at a public square, admitted to an orphanage and then given to guardianship to a couple. However, the search performed by their grandmothers throughout the whole region resulted in their location a year later. A publict conflict emerged between the two families over the legitimacy of the adoption or restitution. This allowed the intervention, not only of military authorities from the involved embassies but also of different local representatives of international organizations as part of a regional and political debate on "subversion" over the media.
\end{abstract}

Regueiro, Sabina A. "De secuestros y localizaciones de niños y niñas 'desaparecidos/as': los albores del activismo de Abuelas de Plaza de Mayo", en Zona Franca. Revista del Centro de Estudios Interdisciplinario sobre Mujeres, Año XXII, № 23, 2014, pp. 37-45.

Recibido: 12 de agosto 2014 - Aceptado: 2 de octubre 2014 
Thus, the study of this case will contribute to the general debate over the complex relationship between politics, rights, kinship and childhood.

Key words: "Abuelas de Plaza de Mayo" - Women's political activism - Adoption - Kinship Childhood

\section{Introducción}

$\mathrm{E}$ I derecho y las burocracias vinculadas a la infancia y la familia se imbrican de diversos modos en los procesos políticos a través de la acción de diversas organizaciones sociales. En las tramas sociales histórico -específicas del Cono Sur, desde las últimas dictaduras militares hasta la actualidad, se han dado fuertes reconfiguraciones jurídico-burocráticas de las nociones de parentesco e infancia. Así, los sentidos, valores, prácticas y procedimientos referidos a los derechos de los niños deben leerse en clave política.

Este artículo analiza una etapa particular de este proceso, las incipientes formas de activismo de organismos de Derechos Humanos (DDHH) compuestos por familiares de detenidosdesaparecidos, especialmente mujeres, para la restitución de niños y niñas secuestrados/as, a través de la movilización de redes institucionales y sociales de cooperación.

Para iluminar este proceso se focalizará en un caso particular, la primera localización de dos niños acompañada por "Abuelas de Plaza de Mayo"1 que tomó dimensión pública regional. A partir de este estudio de caso, se contribuirá al debate general en torno a la compleja relación entre política, derechos, parentesco e infancia.

En términos metodológicos, el caso fue construido a través de notas periodísticas y material obrante en el archivo de Abuelas de Plaza de Mayo: expedientes judiciales, cartas, informes y denuncias institucionales, entre otros documentos. A la vez, nutre esta descripción analítica una entrevista realizada a Victoria Julien Grisonas, la niña secuestrada junto a su hermano, $\mathrm{y}$, por ende, protagonista central en esta historia ${ }^{2}$.

\section{"Abuelas". Tácticas culturales: entre el derecho y la familia}

Durante el terrorismo de Estado en la Argentina (19761983), y aún antes en menor medida, desaparecieron cerca de 500 niños. Algunos de ellos fueron secuestrados junto con sus padres, pero la mayoría nació durante el cautiverio de sus madres en los Centros Clandestinos de Detención. Abuelas de Plaza de Mayo, desde 1977 hasta el presente, registra 116 casos resueltos de niños/as que habiendo sido apropiados/as principalmente por miembros de las Fuerzas Armadas y de Seguridad o de su entorno, pudieron ser localizados/as.

El análisis de las prácticas de búsqueda de estos niños/as "desaparecidos/as" apropiados/ as durante la última dictadura militar argentina, se enmarca en el estudio de las formas de construcción de parentescos en este contexto de violencia de Estado, a partir de las tramas burocrático-administrativas instrumentadas para su implementación y las estrategias jurídico-políticas desplegadas para la restitución a sus familias (Regueiro, 2012).

La intervención del Estado terrorista en las familias consideradas "subversivas" y la apropiación criminal de los/as niños/as desata las acciones orientadas a la localización, identificación y restitución de los/ as hijos/as pequeños/as de militantes políticos perseguidos y detenidos-desaparecidos. Estas fueron llevadas a cabo por sus familiares, tanto en forma individual como colectiva, nucleados en la organización inicialmente llamada Abuelas Argentinas con Nietitos Desaparecidos, posteriormente Abuelas de Plaza de Mayo.

Las microprácticas llevadas a cabo por estas mujeres incluyeron tanto presentaciones jurídico-administrativas ante las burocracias estatales como en organismos no gubernamentales. Estas vías y tramas institucionales son movilizadas a la par de la activación de relaciones personales, a la luz de las prácticas informales que sostuvieron y acompañaron estos recursos.

Las particularidades del contexto institucional del terrorismo de Estado en el que se desarrollan estas demandas dan cuenta de un orden normativo y de hecho en términos de singulares dualidades, la legalidad e ilegalidad, lo oficial y clandestino, que imprimen su marca al activismo primigenio de Abuelas, e implican un campo específico de posibilidad de ciertas prácticas.

Es en estas tramas burocráticas particularísticas, entre normativas y prácticas oficiales y clandestinas que actuaron estas mujeres en el proceso de búsqueda de sus nietos, manipulando normas, constituyéndose en actoras nuevas, impugnando y resistiendo. El modelo para pensar la relación entre representaciones y prácticas de los sectores populares, que diferencia "táctica" de "estrategia" (De Certeau, 2000; Leschziner y Kuasñosky, 2000) es útil para dar cuenta de las particularidades del proceso micropolítico analizado en el 
período abordado. Las tácticas pueden asociarse a la acción de estos familiares durante la dictadura, determinada por la ausencia de un locus político propio, que los obliga a jugar en un terreno impuesto por la ley de un poder extraño, desplegando el "arte del débil". En cambio, el pasaje a la constitución de estrategias generales describe más cabalmente la acción durante la democracia de organismos con prácticas ya institucionalizadas, cuando empiezan a construir un lugar propio de poder, pudiendo jugar dentro de un esquema formal, que implica incluso la constitución de "campos propios".

En este sentido, es interesante resaltar la dimensión cultural constitutiva de la práctica político-social que llevaron a cabo estas mujeres, la manera en la que comenzaron a realizar la construcción social de este "problema" social (Hacking, 1999), esta "causa" colectiva de acción política (Boltanski, 2000), un "frente discursivo" (Fonseca y Cardarello, 2005): la apropiación criminal de niños y la restitución a sus legítimas familias.

Desde una perspectiva gramsciana (Wright, 1998), la cultura es un proceso activo y disputado de construcción de significado de actores en contextos de relaciones de poder desiguales que tiene efectos materiales concretos. En esta primera etapa, en un momento histórico particular, que iluminaría la acción de Abuelas durante la dictadura, los agentes en coalición luchan por la definición de determinadas categorías clave desde una visión particular del mundo, de la realidad y del deber ser, como la diferenciación entre adopción, abandono y apropiación (Villalta, 2012). Sólo en momentos posteriores, estos significados podrán institucionalizarse y convertirse en ley, como aquella que instru- mentó la creación de la Comisión Nacional por el Derecho a la Identidad, el Banco Nacional de Datos Genéticos, los artículos "argentinos" de la Convención Internacional de los Derechos del Niño, etc. Por último, estos sentidos podrían convertirse en hegemónicos, al conformar una nueva manera de pensar sobre un aspecto de la vida que se extiende a otros dominios: el campo de la infancia en la Argentina no puede entenderse sin las Abuelas, desde las leyes de adopción que incluyen el derecho a la identidad, como las concepciones generalizadas sobre la identidad biológica que dieron como resultado distintas organizaciones del tipo de Raíz Natal $^{3}$. Esta difusión de significados y cosmovisiones en la vida cotidiana puede llevar a su naturalización, a su cristalización en "verdades", si bien ninguna ideología está nunca fuera de disputa.

Existe sólo una autonomía relativa de los procesos generadores de sentido en la política: la cultura forma parte de la multidimensionalidad de las dinámicas de poder. Los sentimientos, las creencias, los significados, los valores son necesarios para la legitimación, el apoyo para, en este caso, llevar a cabo las localizaciones y restituciones, así como para la comprensión de las acciones que la posibilitan. Es por eso que hay que prestar atención a las formas simbólicas de la narración, como el mito de la "salvación" de los niños hijos de "subversivos", como a su clasificación como sujetos pasibles de ser apropiados, y a los sentidos que disputan dicho relato a partir de la activación de redes estructurales, de los resortes del poder político y estatal y de distintos grupos de interés (Alexander, 2000).

Por un lado, en el sistema político moderno los grupos sociales deben defender sus intereses dentro del dominio organizado por el Estado, a través de luchas políticas centradas en categorías legales, politizando así todas las cuestiones sociales (Gledhill, 2000), dado que las decisiones políticas se sirven de la forma regulativa del derecho (Habermas, 1999). En este sentido, los derechos $-y$ fundamentalmente los Derechos Humanos- jugaron un papel central en la fundamentación cultural del reclamo en el mismo acto de constitución de Abuelas como un órgano colectivo en octubre de 1977. Uno de los hechos fundamentales en la cristalización de la organización fue la visita del secretario de Estado del gobierno norteamericano Cyrus Vance en noviembre de ese año en la Argentina, para promover una futura visita de la Comisión Interamericana de Derechos Humanos (CIDH) de la Organización de los Estados Americanos (OEA).

Por otro lado, encontramos un universo de sentidos diferente pero igualmente fundamental y constitutivo de la práctica de búsqueda: aquel correspondiente a los valores primordiales, a los sentimientos, al dolor y a la condición de madres y abuelas, de "familia de sangre", que de manera menos evidente que los derechos, tiene también un status político. Estas cuestiones de orden personal se traducen en prácticas políticas, en demandas públicas, mostrando el poder constructor de realidades de los sentimientos, y de la familia como impulsor de construcción de legitimidad y autoridad para reclamar aquellos derechos a través de la formación de organizaciones (Pita, 2005). Se remarca así la dimensión cognitivo-afectiva, valorativa como motivación para la ac- 
ción política y la movilización social, en tanto las emociones pueden ser vistas como partes constitutivas de la acción, como formas de vinculación entre los sujetos y recursos de acción, contribuyendo a la construcción de identidades sociales (Figari y Scribano, 2009:92).

\section{Secuestro, cautiverio, búsqueda y localización de Anatole y Victoria}

Mario Julien y Victoria Lucía Grisonas eran militantes del Partido por la Victoria del Pueblo (PVP) en Uruguay, donde ambos sufren detenciones estando Victoria embarazada de su primer hijo. Tras la dictadura uruguaya de 1973 se trasladan junto a su hijo Anatole Boris a Argentina, donde en 1975 nace su hermana Victoria Eva, en algún lugar de Buenos Aires. El 26 de septiembre de 1976 se produce un operativo conjunto del Ejército y la Policía Federal en la vivienda familiar. Sus padres habían alcanzado a colocar a los chicos en la bañera para protegerlos del ataque. Sin embargo, según una versión de los hechos que le fue comunicada a Victoria, Anatole sale del escondite y ve caer a su madre herida. Otras versiones dicen que los niños vieron a su padre muerto en la vereda y cómo arrastraban a su madre y la colocaban en el baúl de un auto. Un vecino que fue testigo del episodio pidió quedarse con los niños para entregárselos a la familia, pero los responsables del Operativo no se lo permitieron. Los niños de 1 y 4 años de edad fueron secuestrados junto con su madre y llevados al CCD "Automotores Orletti".

Un mes después, en octubre, fueron trasladados nuevamente a Uruguay a otro CCD, el SID donde, según Victoria, había "una parte dedicada a la guar- dería" ${ }^{4}$, y luego a la casa de un sargento de esa dependencia donde permanecieron, por lo menos, hasta fines de noviembre. Anatole, con sus 4 años, podría reconocer a la gente que había visto en todo este proceso. Tal vez por eso, el 27 de diciembre de 1976 fueron trasladados a Chile, y abandonados en la plaza O'Higgins de Valparaíso, por una represora conocida como "la tía Mónica". Allí les dijeron que se quedaran quietos, que ya volverían por ellos, pero no lo hicieron.

Anatole sabía su nombre y tenía un claro acento argentino, lo cual quedó asentado en el periódico chileno El Mercurio que levantó la noticia el 29 de diciembre de 1976, de dos niñitos abandonados en una plaza. El niño contó también que vivían en una casa grande con una escalera y que iban al jardín con su hermana, que una vez "sintió muchas balas" y que su padre que trabajaba en una oficina fue llevado a la "cárcel".

Según los documentos que le entregó a Victoria la delegada presidencial de $\mathrm{DDHH}$, en su momento representante del Alto Comisionado de las Naciones Unidas para los Refugiados (ACNUR), Belela Herrera, pudo saber que su abuela materna Lucía participó de su búsqueda no sólo aportando desde lo económico, sino también desde sus contactos ya que el abuelo de los niños Anatolius, había sido embajador de Lituania. Luego del secuestro viaja a Argentina pero no obtiene el apoyo buscado. Angélica, la abuela paterna de los chicos, coordinó una serie de acciones para localizar a sus nietos ni bien tuvo conocimiento del secuestro. Dijo haber escrito a todo el mundo cientos de cartas a sus 59 años más que en los 50 años precedentes. A través del ACNUR presentaron un habeas corpus poco después del secuestro para encontrar a la familia y un escrito al presidente de facto. Así también se entrevistó con el jefe del I Cuerpo del Ejército entre 1976 y 1980 , con la cabeza de seguridad en la casa de gobierno y el nuncio papal. También enviaron un pedido de ayuda al Papa; a la reina de Inglaterra; a los reyes de España; al presidente norteamericano; al francés; a la ONU y a todas las organizaciones de DDHH.

Uno de los organismos contactado -que tendría una larga historia junto a las Abuelas de Plaza de Mayo- fue CLAMOR, dependiente del Arzobispado de San Pablo, Brasil. El Comité publicó las fotos de los niños en su revista de DDHH. Éstas fueron vistas por una exiliada chilena en Venezuela, que dijo que creía que los chicos estaban en Chile porque allí los había visto en el diario El Mercurio. Se lo comunican a la abuela, diciendo que iban a investigar si se trataba de sus nietos y en qué situación estaban. Así, con este dato, a través de una trabajadora social, los niños pudieron ser rastreados y el 26 de julio de 1979, casi cuatro años después, su abuela paterna pudo localizar a sus nietos, quienes para ese entonces contaban con 4 y 6 años de edad.

\section{"Somos sus padres": parentescos conflictivos}

Cuando los niños fueron hallados solos en la plaza por los Carabineros, los separaron, Anatole fue ubicado en un hogar de varones y Victoria en uno de niñas, lo cual implicó seguramente un nuevo sufrimiento para los niños. A Victoria la entregan a un matrimonio y a Anatole a una mujer soltera. Más tarde deciden que es inconveniente 
separar a los hermanos, pero ninguna de las dos familias podía hacerse cargo de ambos. Por eso la guardadora de la niña contacta a un colega que sabía que estaba haciendo un tratamiento de fertilidad junto con su esposa y que podría tener interés en adoptar a los hermanos. Así el matrimonio obtuvo la guarda y solicitaron la adopción de los hermanos, conservando sus nombres de pila Anatole y Claudia Victoria, ya que el niño repetía incesantemente que esos eran sus nombres.

Sin embargo, el 26 de julio de 1979, una semana antes de que culminara el proceso de adopción, su abuela paterna logra localizar a los niños y el trámite se detiene. Cuando CLAMOR anuncia el hallazgo de los niños pocos días después, el 30 de septiembre, se desata el debate mediático que refleja la disputa entre las familias poder judicial mediante.

En un primer momento, cuando la abuela se encontró con el padre adoptivo los dos lloraron y se abrazaron. Al principio no les dijeron a los chicos quién era ella, la presentaron como su "tía". Acordaron que podrían pasar las vacaciones con ella en Montevideo pero que primero tenían que acostumbrarse a su presencia. Angélica le dijo a la madre adoptiva que estaba muy agradecida por cómo los había cuidado pero que los chicos debían saber "quiénes son", afirmando "¿Qué le diría a mi hijo si no hago nada por los chicos si reaparece?".

Así y todo el caso se reabre en la justicia de menores de Valparaíso. La abuela viaja a Chile varias veces junto a distintos asesores de organismos internacionales para "bloquear" la adopción, solicitando la devolución de los niños a sus familiares (como la comisión de
DDHH y Amnistía Internacional).

Ella quería llevarlos a Uruguay. Un abogado de la Universidad de Chile solicitó la "restitución" de los niños a sus "abuelas legítimas", tratando de causar el "menor daño" posible. El ACNUR, quien organizó el difícil encuentro de la familia con los guardadores, sostuvo que eventualmente los niños serían devueltos a sus familiares más cercanos.

"Se llamó a mi abuela, vino para Chile, hasta que se hace el primer contacto telefónico, ¿sabe qué?, y fue, atroz, por-para mi viejo, fue atroz, porque era, imagínate (se emociona, Ilora) yo estaba con mis papás... con todo el amor... de sus hijos... y llega mi abuela toda, aguerrida, porque, "estos se apropiaron de mis nietos, más encima tuve que perder a mis hijos, a mi hijo, y más encima no me puedo quedar con ellos" (Victoria)

La reacción de los guardadores no se hace esperar. No sólo siguen solicitando la legalización definitiva del vínculo sino que manifiestan ante la prensa que los "padres" de los niños ya estaban "muertos", afirmación por demás conflictiva dado que su abuela planteaba la esperanza de su retorno. Aseveran "sus padres somos nosotros". Así, según su interpretación, la restitución implicaría una "nueva pérdida de sus padres", lo que les causaría a los niños un "trauma" de por vida. La mujer, docente, resalta que cuando recibió a los chicos suspendió su tratamiento de fertilidad porque pensaron que otro hijo sería "fatal para la estabilidad" de los niños. Describen las comodidades de las que los niños gozan, el afecto que reciben, la "familia normal" que componen. El hombre, un "odontólogo cirujano, subdirector del hospital bronco pulmonar", "una persona muy estimada en esta ciudad" dijo que "defenderá por todos los medios a sus hijos".

Argumentaciones de este tipo, que encontramos usualmente en los legajos de guarda y adopción, especialmente en los informes socio-ambientales, se exponen así públicamente en los medios gráficos. Sin embargo, entraron en la disputa actores y problemáticas que en sentido estricto nada tendrían que ver con cuestiones de tutela. De acuerdo al diario argentino $\mathrm{La}$ Prensa ${ }^{5}$, el hecho se originó en una "batida contra grupos sediciosos": los padres de los niños eran de un "grupo extremista", "tupamaros". Este tipo de afirmaciones llevan también a la abuela a contraargumentar afirmando que su hijo era del PVP, pero que no era un "terrorista", que estaba en "contra de la violencia", que era un "socialista cristiano".

De acuerdo al relato oficial, los niños fueron derivados a un "centro de niños abandonados" y se solicitó información dentro y fuera del país sin obtener respuesta, por lo que se inició el proceso de adopción. Podríamos suponer que, de haberse promovido oficialmente desde la Argentina la identificación de los niños, el nombre de Anatole, por cierto muy particular, hubiera facilitado su identificación.

El embajador uruguayo en Santiago de Chile de la Fuerza Aérea afirmó "hay un poco de exageración", "se buscan otros fines que no son claros", "detrás de todo esto hay un fondo político, sobre todo contra quienes luchamos contra la subversión y la sedición y se trata de implicar a los gobiernos". Asevera que el canciller se enteró por los medios, que los familiares no le pidieron nada, y que "la desaparición de los chicos no fue informada en Uruguay". La representante del ACNUR, desmintió al embajador sosteniendo 
que fueron enviadas fotos de los chicos a todas las embajadas de los países limítrofes para que las publicaran.

Esta politización del caso tuvo su correlato en la vida cotidiana de la familia

"Mis viejos fueron perseguidos por adoptarnos, eso, les llegaban amenazas de muerte, había gen- me imagino que por los dos lados, gente que siempre se mete, y pensó que tenian alguna, que estaban involucrados con la represión, u otros que lisa y llanamente tal, no no, son "hijos de socialistas", y todo porque estábamos en plena dictadura, ¿ah?, la cosa era muy difícil" (Victoria)

Finalmente, según el relato de Victoria, prevaleció la postura de que no sería bueno arrancarlos de sus "nuevos padres", que sería una "segunda pérdida", una "segunda ruptura" en una realidad de niños "abandonados" y "maltratados emocionalmente". Su abuela aceptó que permanecieran en la familia adoptiva, siempre que se mantuvieran los vínculos con la familia de origen. Sin embargo, hubo varios viajes de familiares de Uruguay a Chile, pero de Chile sólo salió Anatole. Volvía de Uruguay muy alterado, según su hermana "los recuerdos del pasado lo ponían en contradicción con la realidad que estaba viviendo". Con la niña menor se guardó el secreto varios años más para su "protección" y evitarle el sufrimiento que padecía su hermano.

"Mi hermano tampoco quería que yo supiera porque él sí se acordaba de todo, ¿ah?, un tema de protección, de salvarme del dolor, mi hermano, cuando llegó a la familia, era muy disruptivo y dijo "mierda, tu no eres mi padre", y cosas así. Por lo tanto entró a psicólogos, y ahí lo, lo ayudaron, y le hicieron olvidar ciertas cosas, para poder, sobrevivir emocionalmente" (Victoria)

Cuando la niña contaba con 9 años, sus padres adoptivos, presionados por su familia de origen, le contaron que era adoptada y que los tíos y abuelos que venían a visitarla "con acento" eran sus familiares biológicos. Primero le habían dicho que sus padres habían muerto en un accidente automovilístico - mito clásico para los hijos de desaparecidos, luego deslizaron otra versión.

"Nosotros no somos tus papitos de verdad, pero so- pero igual lo somos, te queremos muchísimo, y tus papitos les pasó algo malo, por un tema político, ellos murieron, y por eso es que tú estás con nosotros acá", eso fue todo lo que me dijeron" (Victoria).

Victoria dice que en ese momento "no sintió nada". Pero cuando llegó a Uruguay encaró a una de sus tías y le pidió que le contara la "verdad" y por primera vez escuchó a hablar de la represión, la tortura, la muerte, que sus padres eran "desaparecidos".

"Es horrible, desestabilizador, fue como que se había abierto un abismo negro y sin fondo. Recuerdo la sensación física que tenía a medida que iba escuchando la verdad. Pensaba ¡cómo me mintieron con esto! Tenía una sensación de injusticia enorme, de desamparo. Sentía que me taladraban por dentro. Por un lado no quería escuchar y por otro lado necesitaba seguir escuchando." (Carrato, 2009)

Cuando ella contó su novedad a su entorno en Chile, descubrió que todos conocían la historia. Victoria empezó a entender muchas cosas hasta entonces incomprensibles, incluso pequeñeces como cuando una compañera del colegio pre- tendió insultarla diciéndoles "tú eres una huérfana". Debido a las amenazas que sufría la familia la comunidad educativa en pleno conocía la historia por una cuestión de "protección". También pudo entender por qué cuando su hermano se enojaba con la guardadora le decía “¿por qué me lo dices si tú igual no eres mi madre?" y contextualizar la fuerte "angustia de separación" que sufría cuando su madre adoptiva se iba a trabajar.

Ya adulta afirma "la verdad, por dura que sea, sana" (Carrato, 2009). Ahí comenzó todo un nuevo proceso de asimilación de la histórica hasta el momento desconocida y de "sentir que esa familia -la uruguaya- es $m i$ familia".

Años después, en 1995, según el relato en un escrito de un abogado de los jóvenes, ambas familias, la adoptiva y la biológica, encontraron "un modo civilizado" de "dialogar y priorizar el interés de los menores". "La abuela conoció a los padres adoptivos y fue persuadida de que lo mejor para los chicos era quedarse con quienes supieron ganar su cariño y cumplían el rol de padres". En una certificación notarial del año 1991, se deja constancia de qué derechos de los niños se hallaban garantizados: la "salud", el "desarrollo", la "atención material y espiritual afectiva" por parte de un "estable matrimonio" de los padres adoptivos. Pero a la vez, se afirma la "integración de los menores y su actual familia adoptiva con su familia natural o consanguínea", a través de la realización de "visitas periódicas y recíprocas", "ampliando en la conciencia de los niños de su círculo de afectos y familiares".

Finalmente, para culminar el proceso de adopción los jóvenes dieron legalmente su propio consentimiento. 
"A los 13 años mis viejos nos dicen "bueno, ahora sí podemos terminar el proceso por fin, de adopción, pero se requiere de la aprobación de ustedes. Entonces, si ustedes quieren ser Larrabeiti hijos, plenamente nuestros, tienen que firmar y decir que consienten voluntariamente ser hijos de nosotros". Y eso sí lo hicimos, cuando yo tenía 12, 13 años. Y ahí nos llevaron a Playa Ancha, a la jueza, y después re piola, firmar ahí, fue un día alegre" (Victoria).

\section{¿Qué es "lo mejor" para los niños y niñas?}

Anatole nace en Uruguay y la dictadura obliga a sus padres a desplazarse a la Argentina donde nace Victoria. De allí, nuevamente por la acción de las fuerzas represivas, son llevados a Uruguay y después a Chile. Una vez localizados, seguirían este camino del Atlántico al Pacífico en pos de recrear sus relaciones familiares. Pero así como los chicos fueron víctimas de la coordinación regional de las dictaduras del Cono Sur, también fueron localizados gracias a otras redes de relaciones activadas por su abuela junto a otras mujeres que estaban en la misma búsqueda. Personas y organismos regionales e internacionales con presencia local fueron movilizados, concretando la identificación de los hermanos.

El caso fue público desde el primer momento, desde que dos niños fueran hallados en la vía pública, sin tener las características que usualmente ostentaban los "menores abandonados". Su pertenencia de clase evidenciada en distintos atributos los destacó de la población común de niños "expuestos": por ejemplo porque estaban "muy bien vestiditos" (Victoria). Esto puede haber sido clave para su localización, porque habría motivado la publicación del caso. Pero lo que fue indispensable para su resolución sin dudas fue la amplia difusión de la desaparición de los niños por parte de CLAMOR, quien a través de su revista llegó hasta Venezuela, país donde inesperadamente se hallaba la respuesta a la incógnita de dónde se encontraban los chicos.

El impacto público del caso se multiplica tras su localización -en parte porque hacia mediados de 1979 la dictadura argentina empieza a resquebrajarse lentamente- mostrando cómo su importancia atraviesa el "campo de la minoridad", por tratarse de hijos de una pareja desaparecida. Esto hace que funcionarios destinados a las relaciones internacionales como embajadores y cancilleres deban pronunciarse al respecto, encarnando la postura política de las dictaduras por ellos representadas.

Este caso representa uno de los primeros capítulos de la incipiente organización de Abuelas de Plaza de Mayo. De hecho, es la abuela paterna de los niños más que el organismo quien mayor protagonismo tiene en esta historia. Desde su dolor y amor de madre y abuela, junto a otras, fue tejiendo este activismo, a partir de creativas microprácticas políticas y de novedosas tácticas que fueron nutriendo innovadoras estrategias que terminaron conformando una auténtica tradición de búsqueda de los niños desaparecidos. Así, las Madres y Abuelas, a partir de la obligación esencial de la maternidad, con aparente estilo apolítico, "por el lado del revés feminista demostraron que lo personal es político" (Barrancos, 2007:268).

Las Abuelas tanto en forma individual como colectiva, apelaron no sólo a instituciones estatales e intergubernamentales, locales e internacionales: en la
Argentina, realizaron la presentación de habeas corpus ante la justicia federal, se interpeló al presidente de facto, y a otros miembros del aparato represivo y eclesiástico asociado. Pero también, se llevó a cabo la radicación de denuncias ante el ACNUR y otras áreas de la ONU; el Vaticano; diversas autoridades gubernamentales y reales; y medios gráficos. Así y todo, fue en las organizaciones de DDHH donde se halló la solución definitiva. A través de la conexión con CLAMOR se activaron redes personales, políticas, informales que fueron determinantes para localizar a los chicos. Fue gracias a una trama de solidaridad tejida regionalmente que se pudo revertir el objetivo del terrorismo de Estado al sacarlos del país: separar a esos niños de sus familias, cubrir el crimen cometido contra sus padres.

De esta manera se pone de manifiesto el doble cariz del terrorismo de Estado con el que tuvo que lidiar este primigenio activismo de los familiares. Por un lado, lo oficial y legal de facto, que niega conocer el destino de los niños e incluso haber recibido las denuncias realizadas, a través de su poder judicial y de su poder ejecutivo. Por otro lado, lo clandestino e ilegal, donde ocurren los hechos, donde niños pequeños son secuestrados, mantenidos en cautiverio en los CCD, trasladados de país en país y finalmente abandonados a su suerte.

En el despliegue de estas tácticas, exitosas como en este caso, se hace sentir el poder de aquellas mujeres supuestamente débiles, en un contexto totalmente adverso, donde se fueron dando los primeros pasos para la construcción de la desaparición y apropiación de niños como un "problema social", una 
"causa colectiva" y un "frente discursivo".

Probablemente, si estuviéramos analizando un conflicto desatado posteriormente a la Convención Internacional sobre los derechos del Niño de la ONU (1989) este apartado final se llamaría "¿Cuál es el interés superior de los niños"? en vez de "¿Qué es lo mejor para los niños?". Pero ambas expresiones comparten su condición de cáscara vacía, ya que poseen una significación indeterminada abstractamente, un contenido vago, variable, sujeto a interpretaciones contradictorias, arbitrarias, que dependen del contexto sociocultural, los valores y el poder discrecional del intérprete. Las categorías utilizadas para definirlo tienen el mismo problema: "salud", "responsabilidad", "familia apropiada", etc., todas deben ser definidas en cada caso concreto (Cardarello, 2007).

Este caso en particular les dará una lección muy importante a las Abuelas: la "localización" de los niños no siempre significará su "restitución". La lucha por volver a llevar a los niños al seno de la familia de origen comienza con la identificación de los chicos. Es una pelea más que hay que dar en el plano cultural, disputando sentidos, creencias, valores vinculados a la infancia y la familia en la arena del derecho. De esta manera, se pone en evidencia una vez más que el parentesco y la identidad son artefactos jurídicos: ¿Quiénes son esos niños? ¿Quiénes son sus padres? Sólo la justicia puede consagrarlos como hijos de unos u otros padres, más allá de quiénes sean los progenitores; autorizar su crianza y pertenencia a una familia u a otra; otorgarles un nombre u otro.

Y es aquí donde cobra importancia la pregunta que parecería orientar las acciones de todos los agentes involucrados en decidir el destino de estos niños ¿Qué es lo mejor para ellos? Así, entran en juego una serie de categorías cuyos sentidos se disputan, negocian, resignifican. ¿Los niños deben estar con su abuela "legítima", de origen, biológica? De este grupo familiar provienen sus padres secuestrados categorizados por los representantes de la dictadura como "sediciosos, extremistas, subversivos" en su condición de militantes "tupamaros" ¿Son éstos considerados implícitamente -en este caso, explícitamente en muchos otros- como padres "negligentes", que "abandonaron" a sus hijos y los pusieron en "peligro"? ¿Deben permanecer con sus guardadores? Este matrimonio es descripto en cambio como profesionales, "estimados" y respetados socialmente, quienes les ofrecen a los niños "estabilidad", "comodidades", o como se expresó posteriormente, promueven su "desarrollo" y "salud", así como proveen "atención material y afectiva".

La "salud", el "daño", el "trauma" son términos del campo médico y "psi", cuyos agentes se constituyen en los tradicionales peritos de los juzgados de menores, contribuyendo con sus teorías científicas diversas a desentrañar qué es "lo mejor" para los niños, instruyendo las decisiones de los jueces ${ }^{6}$. La separación de los niños de sus padres les ha generado un "trauma", ¿lo hará también la restitución? ¿O crecer lejos de su familia de origen? ¿Es más saludable la "verdad" aunque ésta sea cruenta o el "secreto"?

Estas preguntas primeras estarán presentes a lo largo de todas las batallas judiciales que muchas veces son también públicas/mediáticas- llevadas a cabo por las Abuelas, y en torno a ellas fueron tejiendo sus estrategias jurídico-políticas para lograr la restitución de sus nietos. En este movimiento, argumentaron y demostraron que los niños no fueron "abandonados" por sus familias sino que eran buscados, por lo que no necesitaban ser "salvados"; que sus padres no eran demoníacos "terroristas" sino militantes políticos desaparecidos; que si los chicos se hallaban "desamparados" era por el accionar de las fuerzas represivas; y que la restitución es reparadora del "trauma" causado por el secuestro, la violencia vivida, el "secreto" y la "mentira".

Todas estas ideas nos llevan una vez más en el debate socialdisciplinar a afirmar que no hay parentesco pura y simplemente "natural". La relación biológica de los nietos con la abuela no fue suficiente para lograr su restitución. En nuestras sociedades contemporáneas múltiples nociones de familia están detrás de las decisiones judiciales, cuyas reglas elásticas e interpretables terminan adaptándose a distintas realidades. Esto es particularmente evidente en caso atípicos como estos, y por lo tanto, los desenlaces han sido diversos. La adopción podría no haberse consumado pero la discusión en torno a la filiación de los niños se determinó finalmente a partir de una interpretación particular de las normas con el trasfondo de un debate sobre nociones fundamentales en torno a la familia y la niñez.

Este primer caso de impacto regional y público, como parte de la larga historia de Abuelas de Plaza de Mayo, contribuye a la comprensión de la historia de las políticas públicas sobre la infancia, no sólo a nivel local, sino internacional. Prueba de esto es la inclusión de los llamados "artículos argentinos" $\left(7^{\circ}, 8^{\circ}\right.$ y $11^{\circ}$ ) promovidos por las Abuelas 
en la Convención Internacional sobre los Derechos de los Niños, aprobada por la Asamblea General de la ONU en 1989, a la cual adhirieron numerosos países de todo el mundo (la Argentina en 1994 la integra a su Constitución Nacional), consagrando así el "derecho a la identidad".

Notas:

1. http://www.abuelas.org.ar.

2. Entrevista realizada por la autora el 09/10/2008 en la sede de Abuelas de Plaza de Mayo, Capital Federal.

3. Véase http://www.raiznatal.com.ar.

4. Siempre y cuando no se indique lo contrario, el relato de Victoria es producto de la entrevista citada al comienzo de esta ponencia.

5. 03/08/1979. La Prensa. "Esclarecimiento del caso de dos niños abandonados en Chile".

6. Estos agentes de "ortopedia social", de control individual, constituyen un saberpoder que determinará qué es normal, qué es salud y qué no (Foucault, 2003). De acuerdo a la visión "científica" particular de los pediatras y psicólogos, y en función de lo que de sus evaluaciones recuperen los jueces, se dará contenido a las ambiguas categorías en juego. A esta elasticidad se suma el hecho de que al tratarse de un caso considerado "inédito" por tratarse de hijos de desaparecidos, en ocasiones, los profesionales de la salud mental, no encontraron acuerdo

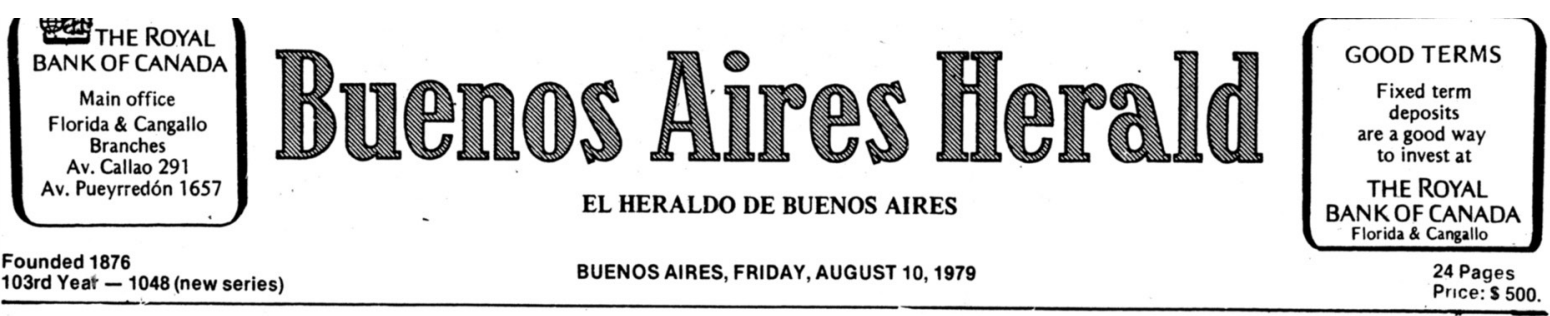

\section{'I knew God would not abandon them'}

\section{Grandmother of misssing}

\section{children never lost hope}

THE grandmother of two tiny children who disappeared after a presumed police raid on their parents' home in San Martín, ver gave up hope that she would find them one day.

In an exclusive interview with the Herald yesterday, Mrs Maria Angelica Cáceres de Julien, said: "You see, I'm a Catholic. I knew that God would never abandon two innocent children."

Mrs Julien, who is in Bueno Aires to legalize documents so that she can clear up the last details in reclaiming her two grandchildren from their foster parents, described her joy when she was Vinted win "Little Anatole came up to when his foster parents introduced me as their aunt. He gave me a hug and then kissed the crucifix I always wear round me neck; asked his foster mother if he knew
God's blessing after dedicating two years of her life to the search for her grandchildren.

"I have writen hundreds of let ers," she said. "I was 59 the day before yesterday and I've writte more letters in the past two yea than I have in all the other 50 ."

When she heard that her son, Mario Roger Julieñ Cáceres, her daughter-in-law Victoria Lucia Grisonas de Julien and their children, Anatole Boris, who was born on September 19, 1972 in Montevideo and Victoria Eva, who was born on May 7, 1975 in Buenos Aires, had disappeare after what was reported in newspapers in Buenos Aires as a police raid on their house in San Martín she began her one woman battle o get back her grandchildren. believed to dave and one of them was reported killed in the raid on the house.

"I've written to everyone," the President of France, $\mathrm{Mr}$ Waldheim of the UN, all the human rights organizations. Oh Wan rights organizations. aders Digest.'”

In Buenos Aires, she followed he sad footsteps, of the many thousands of other people seekin missing relatives. She saw Genera Suárez Mason, the head of security at Government House, the Papal Nuncio - everyone and anyone who might help.

In the hundreds of letters she sent round the world, she always included photographs of the children.

Two months ago, unbeknown to Mrs Julien, her efforts began to pay off. A human rights magazine called Clamor, which is phots

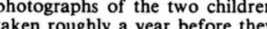
vanished. Refugees from Chile living in . Refugees from Chile lichildren as those they saw in

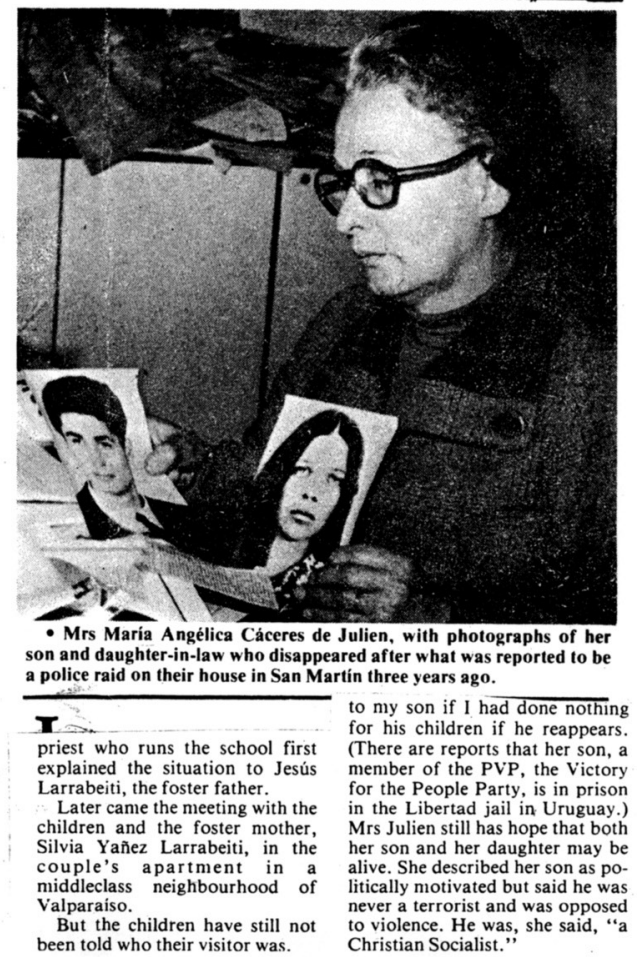


fácilmente. En estos debates el peritaje, considerado científico, sirve como una "sentencia virtual", ofrece "la convicción al magistrado, funda las razones de la sentencia": "el perito sería un agente que reúne las condiciones de testigo, de árbitro y de Juez" (Salessi, 1995:127).

\section{Notas periodísticas:}

1\%08/1979. Buenos Aires Herald. "Niños desaparecidos aparecen en Chile" (traducción propia).

1\%08/1979. El Día. "Hallan en Chile a los hijos de una pareja secuestrada en Argentina".

02/08/1979. Buenos Aires Herald. "Nuestra felicidad está colapsando. Guardadores pelean por los chicos". (traducción propia).

03/08/1979. La Prensa. "Esclarecimiento del caso de dos niños abandonados en Chile".

19/08/1979. Buenos Aires Herald. "Abuela de chicos desaparecidos nunca perdió la fe".

CARRATO, Víctor (2009). "La verdad sana por dura que sea" (entrevista con Victoria Julien Grissonas). La República. $1^{\circ}$ de marzo. Recuperado el 28 de febrero de 2012, de http://www.Ir21.com.uy/politica/354607-la-verdad-sana-por-dura-quesea.

\section{Bibliografía:}

ALEXANDER, Jeffrey (2000). "La preparación cultural para la guerra: código, narrativa y acción social". En Sociología cultural. Formas de clasificación en las sociedades complejas. Barcelona: Anthropos.

BARRANCOS, Dora. (2007). Mujeres en la sociedad argentina, Editorial Sudamericana, Bs. As.

BOLTANSKI, Luc. (2000) El Amor y la justicia como competencias, Ed. Amorrortu, Buenos Aires.

CARDARELLO, Andrea. (2007) "L'intérêt de l'enfant et «l'intérêt de la société » ». (Chapitre 3) En «Trafic légal d'enfants: la formation d'un mouvement de familles pauvres contre les politiques de l'adoption au Brésil ». Thèse de doctorat soutenue au Département d'anthropologie de l'Université de Montréal, Québec, Canada.

DE CERTEAU, Michel. (2000) La invención de lo cotidiano. Artes de Hacer (Tr. Alejandro Pescador), UIA/ITESO.

FIGARI, Carlos y Adrián SCRIBANO (2009) Cuerpo(s), Subjetividad(es) y Conflicto(s). Hacia una sociología de los cuerpos y las emociones desde Latinoamérica, Ediciones Ciccus, FLACSO, Buenos Aires.

FONSECA, Claudia y Andrea CARDARELLO (2005) "Derechos de los más y menos humanos". En Sofía Tiscornia y María Victoria Pita (editoras) Derechos humanos, Tribunales y policías en Argentina y Brasil, Buenos Aires: Ed. Antropofagia.

FOUCAULT, M. (2003) [1978] La verdad y las formas jurídicas, Gedisa, Barcelona.

GLEDHILL, John (2000) El poder y sus disfraces, Bellaterra, Barcelona.

HABERMAS, Jürgen (1999) "Las luchas por el reconocimiento: los fenómenos y los niveles de su análisis". En La inclusión del otro. Estudios de teoría política. Paidós

HACKING, lan (1999) The social construction of what?, Harvard University, Cambridge.

LESCHZINER, Valeria y Silvia KUASÑOSKY (2000) "Cultura y pobreza. Aportes para el estudio de la práctica de los sectores populares", en Cuadernos de Antropología Social No 11. ICA - FFyL-UBA. P. 327-343

PITA, María Victoria (2005) "Mundos morales divergentes. Los sentidos de la categoría de familiar en las demandas de justicia ante casos de violencia policial". En Derechos Humanos, tribunales y policías en Argentina y Brasil, Buenos Aires: Antropofagia.

REGUEIRO, Sabina (2012) Apropiación de niños, familias y justicia. Argentina (19762012), Editorial Prohistoria, Rosario.

SALESSI, Jorge (1995) Médicos maleantes y maricas: higiene, criminología y homosexualidad en la construcción de la nación argentina (Buenos Aires, 1871-1914), Beatriz Viterbo, Rosario.

VILLALTA, Carla (2012) Entregas y secuestros. El rol del Estado en la apropiación de niños, Editores del Puerto, CELS, Buenos Aires.

WRIGHT, Susan (1998) “La politización de la 'cultura'”, en Anthropology Today Vol. 14 No 1, Febrero. Traducción de Florencia Enghel y revisión técnica de Mauricio F. Boivin y Julieta Gaztañaga. 